ORIGINAL ARTICLE

\title{
Prevalence of temporomandibular dysfunction in a group of scuba divers
}

\author{
R D Aldridge, M R Fenlon
}

See end of article for authors' affiliations

......................

Correspondence to: Dr Aldridge, Prosthetics Department, Kings College London, Floor 20 Guys Tower, London Bridge, London SEl 9RT, UK: richardaldridge@ bsac.co.uk

Accepted 25 March 2003
Br J Sports Med 2004;38:69-73. doi: 10.1136/bjsm.2003.005025

Background: Temporomandibular dysfunction (TMD) has been reported to be a common problem in divers, with a prevalence of up to $68 \%$. No evidence for this is available.

Objective: To investigate the prevalence of TMD in divers.

Method: Sixty three subjects were asked to retrospectively complete a questionnaire on symptoms of TMD after diving in warm and cold water areas and in daily life.

Results: The prevalence of TMD was greater in female divers. The prevalence of TMD while diving was about $26 \%$, comparable to that experienced in daily life.

Conclusion: Improvements in mouthpiece design and lighter demand valves mean that TMD is now probably exacerbated by diving rather than caused by it.
$\mathrm{D}$ iving is one of the most rapidly growing adventure sports in the United Kingdom. ${ }^{1}$ Breathing apparatus uses pressurised air, which is reduced to the ambient pressure of the surrounding waters by a regulator valve mounted on a tank. Air is fed to the diver through a flexible hose with a demand valve on the end. The demand valve is held in the mouth by a mouthpiece. The lips create an air tight seal around the mouthpiece preventing water influx. ${ }^{2}$

Most diving related research investigates the hyperbaric conditions experienced when diving. This has enabled divers to increase dive times and depths. More recent research has looked into the equipment used by divers. Commercial mouthpieces have previously been fabricated from injection moulded rubber. Newer mouthpieces are made from silicone or soft acrylic resins.

Mouthpiece designs have a number of common features:

- air intake hole that also attaches to the demand valve

- intraoral screen providing an air/water seal

- lingual flange to provide mouthpiece stability

- interdental bite lugs providing retention. ${ }^{3}$

It has been widely reported that the gripping action on a mouthpiece may result in pain in the masticatory musculature and temporomandibular joint (TMJ) (see the research letter by Balestra et al on p??) known as temporomandibular dysfunction (TMD). New mouthpieces, such as the Sea Cure Custom Mouthpiece, have been designed to overcome the complications of TMD. Manufacturers now incorporate the new design features into mouthpieces fitted on new regulators. Little research is available to show if the new mouthpieces have reduced the prevalence of TMD.

Diving associated TMD is thought to result from forward posturing of the mandible and clenching of the mouthpiece. This results in a lack of posterior occlusal support and uneven loading of the TMJ and masticatory musculature. Cold water is thought to impair the ability of the lips to grip the mouthpiece, forcing reliance solely on the teeth to retain the mouthpiece and thus the demand valve. ${ }^{3}$ Divers also have a tendency to bite harder on one side, again exacerbating the problem. ${ }^{5}$ The pain is sometimes sufficiently severe for dives to be aborted as a consequence. This pain is one of the components of "divers mouth syndrome". ${ }^{6}$
The human musculature is not adapted functionally or anatomically to cope with the extended periods of isometric muscle contraction required to retain the demand valve. Local inflammation of the TMJ has been attributed to the use of demand valve mouthpieces. This may result in blockage of the eustachian tube and possibly labyrinthine dysfunction and associated vestibular disturbances, such as vertigo and disorientation..$^{7-9}$ Obviously these are potentially hazardous underwater.

The signs of TMD vary from one patient to another. Reported findings include ${ }^{10-12}$ :

- pain in the TMJ and ears

- TMJ clicking or crepitus

- trismus and impaired TMJ mobility

- headache and facial pain

- masticatory muscle pain

- stuffy sensation in the ears

- eustachian tube dysfunction

- dizzy spells

It has been suggested that $68 \%$ of dental pain associated with diving can be attributed to $\mathrm{TMD}^{7}$ It has been long recognised that diving can aggravate pre-existing TMD, producing major symptoms in previously symptom free divers. $^{79} 101314$

As TMD is a major complication associated with scuba diving, research has been carried out into mouthpiece design to minimise these complications. This ${ }^{56}$ has concluded that mouthpieces should have the following features:

- interdental bite platform less than $4 \mathrm{~mm}$ thick and $8 \mathrm{~mm}$ wide gripped between first molar and second premolar teeth

- intraoral screens contoured to the shape of the incisors, not extended more than $2 \mathrm{~mm}$ behind the bite blocks

- the screen should be notched to prevent muscle and frenum impingements

- constructed from silicone rather than rubber

Abbreviations: TMD, temporomandibular dysfunction; TMJ, temporomandibular joint 
Please fill in the following spaces and circle the appropriate answers. All data collected is confidential.

$\begin{array}{lllllll}1 \text { Age } & 11-20 & 21-30 & 31-40 & 41-50 & 51-60 & 60+ \\ 2 \text { Gender } & \text { Male } & \text { Female } & & & & \end{array}$

3 Do you feel pain in your jaw muscles or jaw joint? Yes No (Go to Q7)

4 Do you feel the pain: Very often Quite often Sometimes Rarely?

5 Is the pain: Mild Moderate Severe?

6 Is the pain: Present upon waking In the morning Afternoon Evening?

7 Do you have any difficulty opening your mouth as far as you wish because of joint pain or jaw locks? Yes No (Go to Q9)

8 Does this occur: Very often Quite often Sometimes Rarely?

9 Does your jaw click when you eat or open your mouth wide? Yes No (Go to Q11)

10 Does this occur: Very often Quite often Sometimes Rarely?

11 Do you grind or clamp your teeth? Yes No (Go to Q13)

12 Does this occur: Very often Quite often Sometimes Rarely?

13 After you have slept, do you feel well rested? Yes (Go to Q15) No

14 Do you sleep poorly due to: Not sleeping long enough Medication

Pain Distress or anxiety

15 Within the last year have you been treated for jaw pain, difficulty with jaw opening, jaw clicking or popping? Yes No

16 Date of last dive / /

17 Location of dive

18 Estimated temperature of the water

19 How long was the dive? Minutes

20 Did you find the dive stressful? Yes No

21 On a scale of 1-10 give your mouthpiece a rating. $\quad(1=\mathrm{V}$. bad, $10=\mathrm{V}$. good)

22 Is your mouthpiece a standard regulator mouthpiece or is it a custom made mouthpiece? Yes No (Specify

23 Do you have any missing teeth? Yes No

24 Do you wear a denture? Yes No (Go to Q26)

25 If so were you wearing it during the dive? Yes No

26 Did you experience any jaw muscle or joint pain associated with the dive?

27 Did you have any problems opening your mouth as far as you wished during the dive due to joint pain or jaw locks following the dive?

28 Did your jaw click when you ate or opened your mouth following your dive?

29 Did you experience a headache associated with your dive?

30 Did you experience nausea associated with your dive?

31 Do you have stiff shoulders associated with your dive?

Yes No

32 Did you have a sore mouth associated with your dive?

33 Did you have any pain in your ears associated with your dive? Yes No

34 Did you experience any dizziness associated with your dive? Yes No

Additional comments:

Thank you very much for you time.

Figure 1 Questionnaire designed to ascertain the prevalence of temporomandibular dysfunction in a population of divers. 


\begin{tabular}{|c|c|c|c|}
\hline & Cold & Warm & Total \\
\hline Men & 12 & 29 & 41 \\
\hline Women & 11 & 11 & 22 \\
\hline
\end{tabular}

Many papers have described the disorders of the TMJ in relation to scuba mouthpieces. ${ }^{8} 9^{13}$ The prevalence of TMD in divers has been quoted to be between $24 \%{ }^{15}$ and $68 \% .^{7}$ In view of this, we carried out a study to determine if a population of divers exhibited symptoms of TMD at a level comparable to that quoted in previous studies despite improvements in mouthpiece design. The prevalence of divers exhibiting any symptoms of TMD was also determined within the population. The following hypotheses were tested:

(1) Divers do not exhibit significantly more symptoms associated with TMD when diving than in their daily lives.

(2) The prevalence of TMD today is lower than that quoted by previous papers.

\section{METHODS}

\section{Population and location}

Data were collected retrospectively from a sample population of divers asked to complete a questionnaire anonymously, consisting of 34 questions primarily with tick box answers. The sample of divers came from those attending King's College London Sub Aqua Club dive leader training trips and divers diving with Cook Island Divers and Aitutaki Scuba, both in the Cook Islands. Cold water data were collected from divers in the United Kingdom, and warm water data from those in the Cook Islands.

\section{Questionnaire}

The questionnaire (fig l) contained direct questions on symptoms of TMD that affected daily life and similar questions on diving related TMD symptoms. Where participants gave a positive response for a TMD symptom in daily life, they were asked to indicate how often they were affected. Factors such as sex, age, stress, parafunctional behaviour, diving location, and water temperature were included. Divers were also asked to give their mouthpiece an overall rating from $1-10$.

\section{Data analysis}

The results obtained were tabulated on an Excel spreadsheet. $\chi^{2}$ test was used to investigate associations between different variables. The prevalence of TMD symptoms was also calculated.

Table 2 Number of symptoms reported during daily life versus number of divers reporting symptoms

\begin{tabular}{ll}
\hline No of symptoms & No of divers \\
\hline 1 & 18 \\
2 & 12 \\
3 & 4 \\
4 & 1 \\
\hline
\end{tabular}

Table 3 Number of symptoms associated with diving versus number of divers reporting symptoms

\begin{tabular}{ll}
\hline No of symptoms & No of divers \\
\hline 1 & 16 \\
2 & 8 \\
3 & 4 \\
4 & 1 \\
5 & 0 \\
6 & 0 \\
7 & 1 \\
\hline
\end{tabular}

\section{RESULTS}

In total, 63 sets of data were collected, 41 from male divers and 22 from female divers. More data were collected for the former in both warm and cold water sites (table 1).

Divers were aged 11-40. More of the older divers were found in the warm water sites, with no data from divers over 30 in cold water locations.

A number of divers reported symptoms related to TMD in day to day life (table 2).

Fewer divers reported symptoms of TMD associated with diving compared with daily life. However, divers reporting symptoms of TMD were not always the same in both groups (table 3).

The diving associated prevalence of one TMD symptom for our population of divers was $47.6 \%$. Surprisingly the nondiving associated TMD prevalence was $55.6 \%$ in our population. However, TMD should really be defined by the presence of more than one symptom. If two symptoms are required for diagnosis, the prevalence was $22.2 \%$ and $27 \%$ respectively. If three symptoms are required, the prevalence was $9.5 \%$ and $7.9 \%$ respectively. It is only therefore when three symptoms are required to diagnose TMD that more symptoms are reported when diving. This assumes that all symptoms are awarded the same importance and that symptoms reported are solely TMD in origin (table 4).

If the cold and warm water data are taken separately, it is clear that the prevalence of TMD is higher in the cold water divers (tables 5 and 6 ).

Most divers were happy with their mouthpieces, with the mode figure for mouthpiece rating being 8 out of a possible 10.

The $\chi^{2}$ tests showed an association between sex and limitation of mouth opening after diving. Limitation of opening is a widely accepted symptom of TMD. ${ }^{10}{ }^{11}$ This result supports previous research. ${ }^{16}$ Four women but no men reported limitation of mouth opening when diving (table 7).

An association was also found between sex and divers reporting nausea. Four female but just one male diver reported nausea after diving (table 8 ).

More divers reported jaw stiffness in cold than warm water. An association was found between jaw stiffness and

\begin{tabular}{|c|c|c|}
\hline \multirow[b]{2}{*}{$\begin{array}{l}\text { No of symptoms } \\
\text { reported }\end{array}$} & \multicolumn{2}{|c|}{ Prevalence of TMD (\%) } \\
\hline & Non-diving related & Diving related \\
\hline $\begin{array}{l}1 \\
2 \\
3\end{array}$ & $\begin{array}{l}55.6 \\
27 \\
7.9\end{array}$ & $\begin{array}{l}47.6 \\
22.2 \\
9.5\end{array}$ \\
\hline
\end{tabular}


Table 5 Number of symptoms reported in divers in cold and warm water areas

\begin{tabular}{lll}
\hline No of symptoms & Cold & Warm \\
\hline 1 & 7 & 9 \\
2 & 4 & 4 \\
3 & 1 & 3 \\
4 & 1 & - \\
5 & - & - \\
6 & - & - \\
7 & 1 & - \\
\hline
\end{tabular}

water temperature. Just under 39\% of divers reported some jaw stiffness after diving in cold water whereas just 5\% of divers reported jaw stiffness after diving in warm water (table 9). This again supports findings of previous research. ${ }^{45}$

\section{DISCUSSION}

The spread of data collected for male and female divers was uneven, with more male subjects. This was more pronounced for the cold water data than the warm water data. Historically, scuba diving has been a male dominated sport. This is predominantly still the case especially in cold water areas and is reflected by the data collected.

An association was found between sex and mouth opening and also between sex and nausea. More women than men reported symptoms (tables 7 and 8). This agrees with previous work on TMD which has found that more women suffer from TMD than men. ${ }^{11}{ }^{16}$ This may be due to anatomical differences such as mandibular angulation and masticatory muscle insertion. As more data were collected for male divers, it is possible that the overall incidence of TMD has been underestimated.

The age of the divers studied varied greatly. More older divers were found diving in the warm water areas. This can probably be attributed to the cost of accessing these areas from the United Kingdom and also the time of year at which the data were collected. No data were obtained from divers over the age of 30 in cold water areas.

Jaw stiffness was reported significantly $(p=0.01)$ more often in cold water than warm water areas (table 9). This was expected and agrees with previous research, suggesting that TMD is more of a problem in cold water areas. ${ }^{4}$ However, the data collected in cold water areas were from divers in the age range more likely to suffer from TMD-that is, 20-40. ${ }^{117}$

In general, most participants liked their mouthpiece. Most were of a modern design, although this comes from observation rather than data collected. Surprisingly, only one diver had a customised mouthpiece. This respondent reported no TMD symptoms while diving but did suffer from non-diving associated TMD symptoms. It is possible that the diver's mouthpiece was providing a larger occlusal surface area allowing a reduced force per unit area.

\begin{tabular}{|c|c|c|}
\hline \multirow{2}{*}{$\begin{array}{l}\text { No of } \\
\text { symptoms }\end{array}$} & \multicolumn{2}{|c|}{ Prevalence of symptoms (\%) } \\
\hline & Cold water & Warm water \\
\hline 1 & 58.3 & 40 \\
\hline 2 & 29.2 & 17.5 \\
\hline 3 & 12.5 & 7.5 \\
\hline 4 & 8.3 & - \\
\hline 7 & 4.2 & - \\
\hline
\end{tabular}

\begin{tabular}{|c|c|c|}
\hline & No limitation & Limitation \\
\hline Female & 18 & 4 \\
\hline Male & 41 & 0 \\
\hline Total & 59 & 4 \\
\hline \multicolumn{3}{|c|}{$\chi^{2}=7.9599 ; p=0.005$} \\
\hline
\end{tabular}

These data show that, in this population, more divers reported at least one symptom of TMD during daily life than when diving. This was not the case when diagnosis was based on the presence of three symptoms (table 4). There is no universally agreed number of TMD symptoms required for an accurate diagnosis. As many TMD symptoms are commonly associated with other conditions, at least two or three symptoms are required for a reliable diagnosis. Furthermore, TMD symptoms resulting from diving but manifesting shortly afterwards were probably incorrectly attributed to non-diving activity. Also there were more questions on non-diving associated symptoms on the questionnaire. In this study all reported symptoms were given equal weighting. However, some symptoms are more important for TMD diagnosis than others-for example, jaw stiffness compared with nausea. No attempts were made to differentiate between symptom importance.

The prevalence of TMD in this study varies greatly according to the number of symptoms on which the diagnosis was based. Patients with TMD would probably exhibit multiple symptoms. Those with only one or two symptoms probably did not actually have TMD, especially when diving complications such as nausea are taken into account. They may in fact have had sea sickness or mild decompression illness. The prevalence while diving ranges from $47.6 \%$ of divers exhibiting one symptom to $9.5 \%$ exhibiting three symptoms. While not diving, the prevalence ranges from $55.6 \%$ for one symptom to $7.9 \%$ for three symptoms (table 4 ). However, if the data are separated into those from cold and warm water areas, the prevalence in the warm water group falls to $40 \%$ reporting one symptom and $7.5 \%$ reporting three symptoms. For the cold water group, the prevalence rises to $58.3 \%$ reporting one symptom and $12.5 \%$ reporting three symptoms. One diver in this group reported seven symptoms (table 6). This difference in prevalence between warm and cold water is expected and is supported by the significant association between water temperature and jaw stiffness.

\begin{tabular}{|c|c|c|}
\hline & No nausea & Nausea \\
\hline Female & 18 & 4 \\
\hline Male & 40 & 1 \\
\hline Total & 58 & 5 \\
\hline
\end{tabular}

$\begin{aligned} & \text { Table } 9 \text { Jaw stiffness of diver versus water } \\
& \text { temperature }\end{aligned}$
\begin{tabular}{lcl} 
& Cold & Warm \\
\hline No stiffness & 14 & 38 \\
Stiffness & 9 & 2 \\
Total & 23 & 40 \\
\hline$\chi^{2}=11.8036 ; p=0.001$. & \\
\hline
\end{tabular}




\section{Take home message}

- TMD prevalence in divers is lower than previously quoted and not significantly higher than in the general population

- TMD prevalence is higher in female divers and in cold water areas

- Improved mouthpiece design and reduced demand valve weight are the probable reasons for the reduced prevalence of TMD; mouthpieces are now likely to exacerbate rather than cause TMD

- Divers with symptoms of TMD should seek dental help, possibly in association with the construction of a customised mouthpiece

Other studies have suggested the prevalence of TMD in divers to be between $24 \%{ }^{15}$ and $68 \% .{ }^{18}$ Our data suggest that the prevalence of TMD in this group of divers is lower than previously thought depending on the number of symptoms selected as being diagnostic of TMD. As more than one symptom of TMD is probably required for an accurate diagnosis, this study suggests that the prevalence of TMD is probably $5-30 \%$ for cold water divers and $5-20 \%$ for warm water divers. The prevalence of TMD in daily life in these subjects is about $5-30 \%$. This reflects the results of other studies. ${ }^{17}$ Therefore diving with more modern mouthpieces and lighter demand valves probably does not cause TMD but may exacerbate it, especially if diving is confined to warmer water areas.

Our results suggest that predominantly the same people are affected by TMD while diving and during day to day living. This is supported by the similarity in all prevalence figures for diving and non-diving with differing numbers of symptoms (table 6).

\section{Further research areas}

It is possible that more significant results would have been obtained if a larger number of divers had been studied.
Further research should increase the sample size. A weighting for different TMD symptoms would also be of use. An extension of this research may be to investigate the effectiveness of custom made mouthpieces to reduce TMD in those divers affected.

\section{Authors' affiliations}

R D Aldridge, Kings College London, UK

M R Fenlon, GKT Dental Institute, London, UK

\section{REFERENCES}

1 British Subaqua Club. BSAC annual report and accounts of 1989. British Subaqua Club.Town?

2 Busuttili M, Holbrook M, Ridley G, et al. Sport diving, the British Subaqua Club diving manual. London: Ebury Press, 1998.

3 Newton J, Hobson R, Sturrock K. Design and construction of customised mouthpieces for subaqua diving. Eur J Prosthodon Restor Dent 1995;3:223-6.

4 Jones C, Graham J. Underwater orthodontics. Br J Orthod 1990; 17:325-8.

5 Grant S, Johnson F. Divers mouth syndrome: a report of two cases and construction of customised made regulator mouthpieces. Dental Update July/ August 1998:254-6.

6 Goldstein G, Katz W. Divers mouth syndrome. NY State Dent J 1982;48:523-5.

7 Hobson R. Temporomandibular dysfunction syndrome associated with scuba diving mouthpieces. Br J Sports Med 1991;25:49-57.

8 Pinto O. Temporomandibular joint problems in underwater activities. J Prosthet Dent 1966:16:772-81.

9 Roydhouse N. The jaw and scuba diving. J Otolaryngol Soc Aust 1977;4:162-5

10 Pinto O. Temporomandibular joint problems in underwater activities. J Prosthet Dent 1966:16:772-81.

11 Helkimo M. Epidemiological surveys of dysfunction of the masticatory system. Oral Sci Rev 1976;1:54.

12 Helkimo M. An epidemiological investigation of symptoms of dysfunction in Lapps in the north of Finland. Proc Finn Dent Soc 1974;70:37-9.

13 Storer R, Bowman A. An unusual factor in the disharmony of the masticatory system. Br Dent J 1969;126:80-1.

14 Jagger $\mathbf{R}$, Jackson $S$, Jagger $D$. In at the deep end: an insight into scuba diving and related dental problems for the GDP. Br Dent J 1997;183:10.

15 Roydhouse N. 1001 disorders of the ear nose and sinuses in scuba divers. Can J Appl Sport Sci 1985;10:99-103.

16 Solberg W, Woo M, Houston J. Prevalence of mandibular dysfunction in young adults. J Am Dent Assoc 1979:98.

17 Macfarlane T, Blinkhorn A, Davies R, et al. Orofacial pain in the community: prevalence and associated impact. Community Dentistry and Oral Epidemiology, 2002;30(1):52-60

18 Hobson R. Diving and dental pain S Pac Underwater. Med Soc J 1987; 17:43-6. 